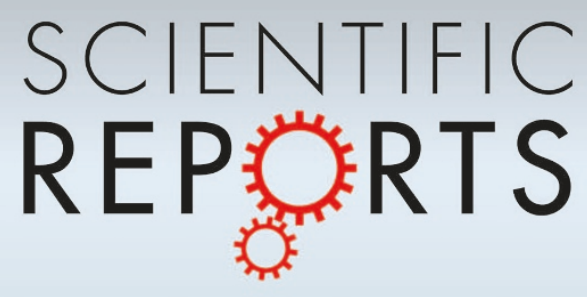

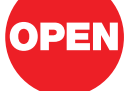

SUBJECT AREAS:

MODELLING AND

THEORY

MAGNETIC MATERIALS AND

DEVICES

APPLIED PHYSICS

MATERIALS PHYSICS

Received

13 December 2011

Accepted

19 June 2012

Published

25 July 2012

Correspondence and requests for materials should be addressed to K.S.K. (stone99.kim@ samsung.com)

* These authors contributed equally to this work.

\section{Current Driven Magnetic Damping in Dipolar-Coupled Spin System}

\author{
Sung Chul Lee*, Ung Hwan Pi*, Keewon Kim, Kwang Seok Kim, Jaikwang Shin \& U-In Chung
}

Samsung Advanced Institute of Technology (SAIT), San \# 14-1, Nongseo-dong, Giheung-gu, Yongin-si, Gyeonggi-Do 446-712, Korea.

Magnetic damping of the spin, the decay rate from the initial spin state to the final state, can be controlled by the spin transfer torque. Such an active control of damping has given birth to novel phenomena like the current-driven magnetization reversal and the steady spin precession. The spintronic devices based on such phenomena generally consist of two separate spin layers, i.e., free and pinned layers. Here we report that the dipolar coupling between the two layers, which has been considered to give only marginal effects on the current driven spin dynamics, actually has a serious impact on it. The damping of the coupled spin system was greatly enhanced at a specific field, which could not be understood if the spin dynamics in each layer was considered separately. Our results give a way to control the magnetic damping of the dipolar coupled spin system through the external magnetic field.

pins misaligned to the magnetic field relax into the field direction by transferring their energy to the environment. The relaxation rate is governed by the magnetic damping constant $(\alpha)$ which reveals the interaction between the spins and the environment around the spins. Though $\alpha$ is a material-specific value, it can be actively controlled by the spin transfer torque ${ }^{1,2}$. The active reduction of $\alpha$ gives such interesting phenomena as magnetization reversal ${ }^{3-6}$ and steady spin precession ${ }^{7-12}$, which have given birth to the novel devices like the spin transfer torque magnetoresistive random access memory (STT-MRAM) ${ }^{13,14}$ and the spin torque nano-oscillator (STNO $)^{15}$. In general, these spintronic devices consist of two main spin layers called as free and pinned layer. Although each layer is coupled to the other by the dipolar interaction and the spin transfer torque, the spin dynamics driven by their coupling has not been considered seriously. Only recently, the spin dynamics coupled by the mutual spin transfer torque has been studied by micromagnetic calculation ${ }^{16,17}$. But the dipolar interaction was assumed to give only marginal effects because the dipolar field from the pinned layer is generally compensated by the antiferromagnetically coupled additional layer, i.e. synthetic antiferromagnet $(\mathrm{SAF})^{17-19}$. Here in this report, however, we show that the dipolar coupling actually has a serious impact on the current driven spin dynamics. We observed that the current driven spin oscillation disappears at a specific magnetic field. The micromagnetic calculation and the numerical estimation of the eigenmodes on the model systems with different dipolar interactions show that the observed behavior originates from the dipolar coupling. As will be described later, the current driven magnetic damping of the dipolar-coupled spin system is closely related to the transfer of energy between the two layers. By changing the energy transfer rate with the external magnetic field, one can adjust the damping of the coupled spin system.

\section{Results}

Anomalous discontinuity in spin oscillation mode observed in magnetic tunnel junction. The sample for our study is a typical magnetoresistive tunneling junction (MTJ), which consists of the bottom layer [Si/SiO2/ $\mathrm{TiN}(60)]$, SAF $\left[\operatorname{PtMn}(15) / \mathrm{Co}_{90} \mathrm{Fe}_{10}(1.5) / \mathrm{Ru}(0.8) / \mathrm{Co}_{40} \mathrm{Fe}_{40} \mathrm{~B}_{20}(1.5)\right]$, tunneling barrier $[\mathrm{Mg}(0.3) / \mathrm{MgO}(0.53)]$, magnetic free layer $\left[\mathrm{Co}_{20} \mathrm{Fe}_{60} \mathrm{~B}_{20}(2.0)\right]$, and capping layer[Ta(5)/Ti(15)/Ru(15)] as shown in Fig. 1. Here the numbers in the parenthesis represent the nominal thickness in the unit of nanometer. In order to study the coupled motion between the free layer (FL) and the top pinned layer of the SAF (TPL), the thickness of the pinned layer was thin enough to be excited by the spin transfer torque. In previous studies that focus only on the dynamics of the FL spin, the thickness of the TPL was much thicker ${ }^{20,21}$ or wider $^{22}$ than the FL, inhibiting the excitation of the TPL. The MTJ cell has a circular shape with a diameter of $90 \mathrm{~nm}$, minimizing the in-plane shape anisotropy. The current driven spin precession yields a resistance oscillation in the circular MTJ cell, which has been measured under different external fields $\left(\mu_{0} H\right)$. The polarity of $\mu_{0} H$ was defined as positive when the magnetization of the FL 


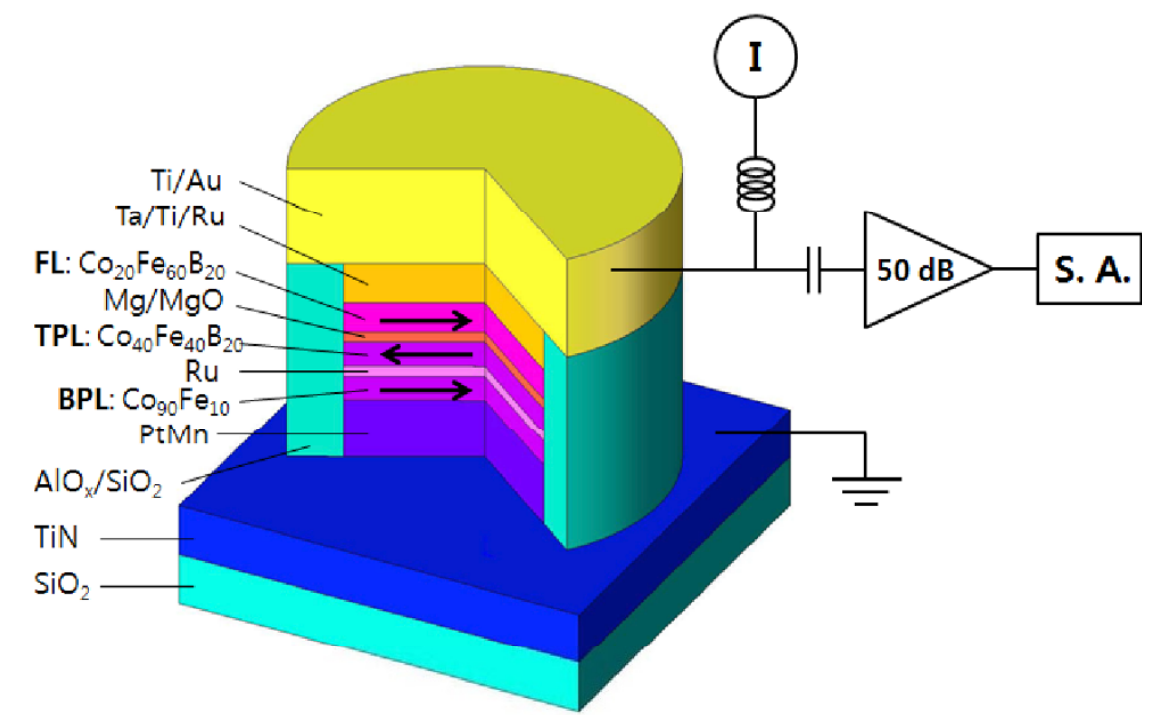

Figure $1 \mid$ Schematic diagram of the MTJ structure and measurement setup. The three ferromagnetic layers are magnetically coupled. The free layer $\left(\mathrm{Co}_{20} \mathrm{Fe}_{60} \mathrm{~B}_{20}\right)$ is dipolar-coupled with the top pinned layer $\left(\mathrm{Co}_{40} \mathrm{Fe}_{40} \mathrm{~B}_{20}\right)$. The top pinned layer is exchange biased antiferromagnetically with the bottom pinned layer $\left(\mathrm{Co}_{90} \mathrm{Fe}_{10}\right)$ through the Ru layer, forming a synthetic antiferromagnet. For the study of coupled spin motion, the thickness of the pinned layer was thin enough to be excited by the spin transfer torque. The current driven spin oscillation yields the resistance change in the MTJ, the microwave by which is measured through the spectrum analyzer (S.A.).

is antiparallel with the TPL. The current bias polarity was defined as positive when the electron moves from the TPL to the FL.

Figure 2a shows the resistance of the MTJ cell as a function of $\mu_{0} H$ in the current density $J=7.8 \times 10^{6} \mathrm{~A} / \mathrm{cm}^{2}$. Under the negative (positive) field, the spins in the FL are parallel (antiparallel) to the spins in the TPL, yielding the lower (higher) magnetoresistance level. The spins in the TPL begin to be tilted from the spin flop-field $\mu_{0} H_{s f}=$ $90 \mathrm{mT}$, where the external field becomes comparable to the exchange field between the TPL and bottom pinned layer (BPL) of the SAF. The alignments of the spins in the three layers are schematically shown in Fig. 2a as arrow marks for the comparison with the resistance curve.

The color-coded power spectrums of the MTJ cell in the current biases $J=+7.8 \times 10^{6} \mathrm{~A} / \mathrm{cm}^{2}$ and $-7.8 \times 10^{6} \mathrm{~A} / \mathrm{cm}^{2}$ are shown in Fig. $2 \mathrm{~b}$ and $2 \mathrm{c}$, respectively. In the field range $0<\mu_{0} H<\mu_{0} H_{s}$, one can observe distinctive dependence of the oscillation mode on the current bias polarity. In positive (negative) current bias, the spins in the FL (TPL) are dominantly excited, yielding a blue (red) frequency shift with the increasing field. Above the spin-flop transition $\mu_{0} H>$ $\mu_{0} H_{s f}$, both the free and pinned layer show blue frequency shifts. These general features agree with the previous reports on the eigenmodes of the FL and TPL in the MTJ cell ${ }^{23-25}$.

There are several interesting points in Fig. $2 \mathrm{~b}$ and $2 \mathrm{c}$. A discontinuity is clearly observed in the oscillation mode. In Fig. $2 b$, the free layer oscillation observed at low fields disappears at around $\mu_{0} H=$ $40 \mathrm{mT}$, and reappears at $\mu_{0} H>60 \mathrm{mT}$. One should note that the point by point discontinuity in the field range $\mu_{0} H<30 \mathrm{mT}$ is just due to the large interval of the sampling fields in our experiment. This is different from the real discontinuity of the oscillation mode.

One can also find a gap in the oscillation frequency at around the disconnected point. The extension of the lower field mode by the Kittel formula ${ }^{26}$ does not matches well with the higher field mode. The oscillation mode of the TPL in Fig. $2 c$ also shows similar discontinuity and frequency gap, which implies that the observed feature is independent of the current polarity. The explanation of the observed anomalous breakdown of the oscillation mode is the main subject of this article.

Micromagnetic calculation : effect of dipolar coupling on spin oscillation. The magnetization dynamics of the coupled spin layers have been calculated using the Landau-Lifshits-Gilbert-SlonczewskiSlonczewski (LLGS) equation given by ${ }^{3,27,28}$

$$
\frac{d M}{d t}=-\gamma \mu_{0} M \times H+\frac{\alpha}{M} M \times \frac{d M}{d t}-\gamma \frac{a J}{M} M \times(M \times p)
$$

where $\gamma, \alpha, a_{J}$, and $p$ represents the gyromagnetic ratio, the intrinsic damping constant, the amplitude of the spin-torque in the unit of magnetic field, a unit vector parallel to the electron spin polarization. In order to study the effect of the dipolar coupling between the layers, the micromagnetic calculations have been carried out on several different separations $(s)$ between the TPL and FL from $0.8 \mathrm{~nm}$ to $50 \mathrm{~nm}$. Although electrons cannot tunnel through the oxide barriers in case of large $s$ value in reality, we assumed the same tunneling current could pass through the MTJ in our calculations.

The color-coded microwave power calculated on the model system with $s=0.8 \mathrm{~nm}, 10 \mathrm{~nm}, 50 \mathrm{~nm}$ under the positive and negative current biases $J= \pm 8.0 \times 10^{6} \mathrm{~A} / \mathrm{cm}^{2}$ are depicted in Fig. $3 \mathrm{a}-3 \mathrm{c}$ and in Fig. 3d-3f, respectively. The case of $s=0.8 \mathrm{~nm}$ shows nearly the same oscillation feature with the experiment. There is a clear discontinuity and frequency gap in the oscillation mode at around $45 \mathrm{mT}$. As $s$ increases, i.e., the dipolar interaction between the FL and TPL becomes weaker, the gap between the higher frequency mode and lower frequency mode decreases. At the separation of $50 \mathrm{~nm}$, the oscillation mode is nearly connected with a negligible gap like the oscillation mode of a 'non-coupled' single spin system. This reveals that the observed breakdown and frequency gap in the oscillation mode at around $45 \mathrm{mT}$ comes from the dipolar coupling between the FL and TPL.

Normal modes of dipolar coupled spin oscillation. To deeply understand the coupled dynamics, we calculated the eigenmodes of the model system by solving the LLGS equation in a small excitation limit. The azimuthal and polar angles of the magnetization in each layer have been defined as $\varphi_{i}$ and $\theta_{i}$, respectively. Here $i$ represents the layer number of the BPL $(i=1)$, TPL $(i=2), \mathrm{FL}(i=3)$. The LLGS equation in Eq. 1 can be described according to the above six variables $\boldsymbol{X}=\left(x_{1}, x_{2}, x_{3}, x_{4}, x_{5}, x_{6}\right)=\left(\varphi_{1}, \theta_{1}, \varphi_{2}, \theta_{2}, \varphi_{3}, \theta_{3}\right)$ in the form

$$
\frac{d x_{i}}{d t}=F_{i}\left(x_{1}, x_{2}, x_{3}, x_{4}, x_{5}, x_{6}\right) i=1 \sim 6
$$



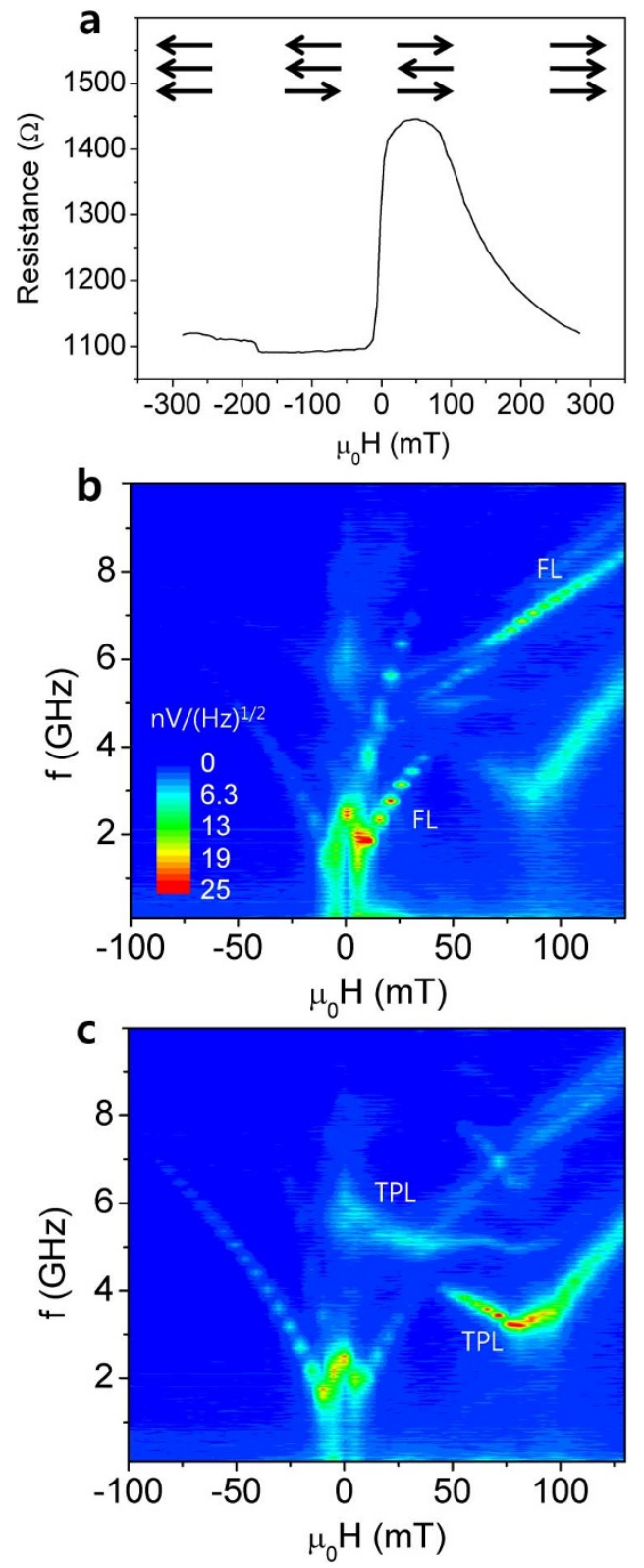

Figure $2 \mid$ Resistance and color-coded microwave power spectrum of the MTJ cell as a function of the magnetic field. (a) Resistance measured in the current density $J=7.8 \times 10^{10} \mathrm{~A} / \mathrm{m}^{2}$. Under the negative (positive) field, the spins in the free layer are parallel (antiparallel) to the spins in the top pinned layer, yielding the lower (higher) magnetoresistance level. The spins in the top pinned layer begin to be tilted from the spin flop field $\mu_{0} H_{s f}=90 \mathrm{mT}$. The alignments of the spins in the three layers are shown as arrow marks for the comparison with the resistance curve. (b),

(c) Color-coded power spectrums of the MTJ cell in the current biases $J=+7.8 \times 10^{10} \mathrm{~A} / \mathrm{m}^{2}$ and $-7.8 \times 10^{10} \mathrm{~A} / \mathrm{m}^{2}$, respectively. (a) In positive current bias, the spins in the free layer are dominantly excited, yielding a blue frequency shift with the increasing field. (b) In the negative bias, the spins in the top pinned layer are dominantly excited, yielding a red frequency shift. A discontinuity in the oscillation mode is observed in both cases at around $\mu_{0} H=45 \mathrm{mT}$, which is about half the spin-flop field.

A linearized form of the above equation around the stationary point $X_{0}=\left(x_{10}, x_{20}, x_{30}, x_{40}, x_{50}, x_{60}\right)$ following $F_{i}\left(X_{0}\right)=0$ can be written as

$$
\frac{d \delta x_{i}}{d t}=\left.\sum_{j=1}^{6} \frac{\partial F_{i}}{\partial x_{j}}\right|_{x_{j}=x_{j 0}} \delta x_{j}
$$
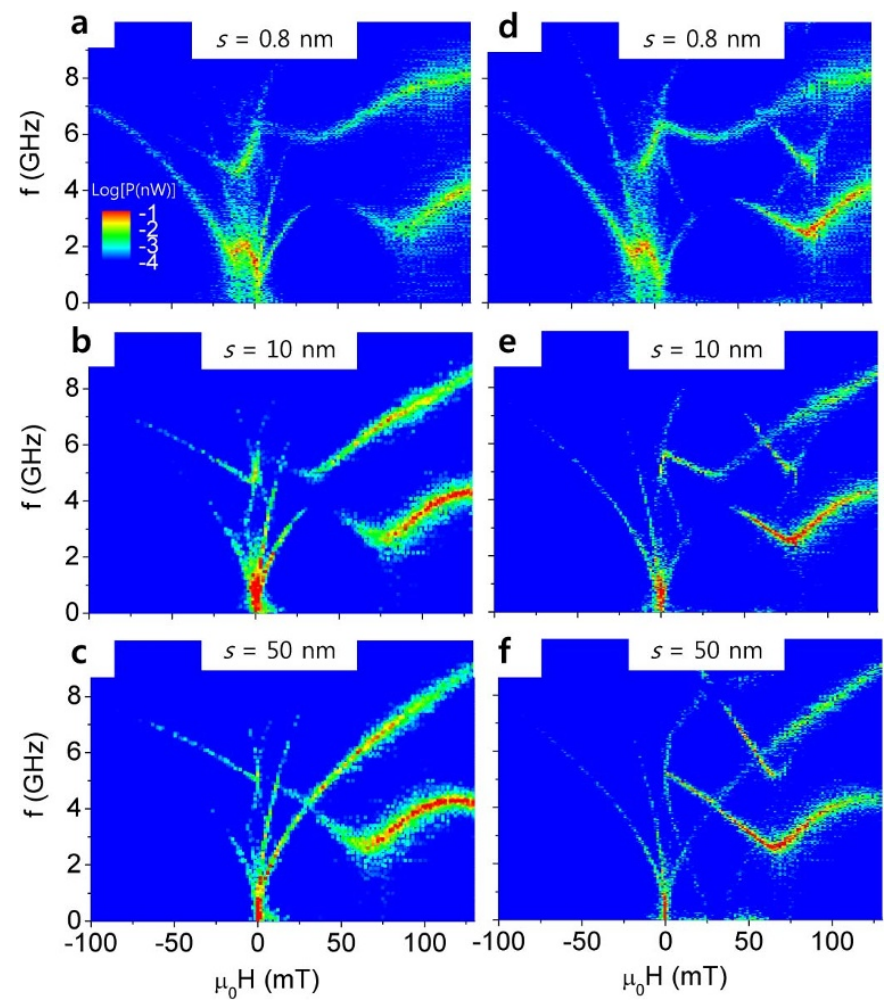

Figure $3 \mid$ Microwave power spectrum calculated by on the model MTJ device under several different separations $(s)$ between the free layer and top pinned layer. (a), (b), (c) Microwave power spectrums as a function of the external field calculated from the LLGS equation under the positive current bias $J=+8.0 \times 10^{10} \mathrm{~A} / \mathrm{m}^{2}$ on $s=0.8 \mathrm{~nm}, 10 \mathrm{~nm}, 50 \mathrm{~nm}$, respectively. (d), (e), (f) The same calculations under the negative current bias $J=-8.0 \times 10^{10} \mathrm{~A} / \mathrm{m}^{2}$ on $s=0.8 \mathrm{~nm}, 10 \mathrm{~nm}, 50 \mathrm{~nm}$, respectively. There is also a clear discontinuity and frequency gap in the oscillation mode at around $45 \mathrm{mT}$. As $s$ increases, the gap between the higher frequency mode and lower frequency mode decreases.

Here $\boldsymbol{\delta} \mathrm{X}=\boldsymbol{X}-\boldsymbol{X}_{\mathbf{0}}\left(\delta x_{i}=x_{i}-x_{i 0}\right)$ represents a small deviation from the stationary point. If the six eigenvalues and eigenvectors of the $6 \times 6$ matrix $\bar{T}=T_{i j}=\left.\frac{\partial F_{i}}{\partial x_{j}}\right|_{x_{j}=x_{j 0}}$ are respectively $\varepsilon_{k}$ and $U_{\boldsymbol{k}}(k=1 \sim 6)$, i.e., $\bar{T} U_{k}=\varepsilon_{k} U_{k}$, then $\delta \mathrm{X}$ in Eq. 3 can be written as

$$
\delta X=\sum_{k=1}^{6} c_{k} \exp \left(\varepsilon_{k} t\right) U_{k}
$$

Here $c_{k}$ is a numerical constant determined by the initial condition of the motion. The motion of each variable is the superposition of the six eigenmodes weighted by $\exp \left(\varepsilon_{k} t\right)$. The imaginary part of the eigenvalue $\operatorname{Im}\left(\varepsilon_{k}\right)$ corresponds to the angular frequency of the oscillatory eigenmode, and the real part $\operatorname{Re}\left(\varepsilon_{k}\right)$ reveals the decay rate of the oscillation. Negative $\operatorname{Re}\left(\varepsilon_{k}\right)$ implies a mode whose oscillation decays out even though it has an initial oscillation amplitude. In the positive $\operatorname{Re}\left(\varepsilon_{k}\right)$, on the other hand, the spin oscillation is feasible to be excited ${ }^{29}$. In this sense, the value of $\operatorname{Re}\left(\varepsilon_{k}\right)$ is the crucial factor that determines the damping rate of the spin oscillation.

The matrix $\bar{T}$ has been numerically calculated under different external fields and current biases from the LLGS equation. It gives six eigenvalues, three of which are the complex conjugates of the others. The three real and imaginary part eigenvalues $\operatorname{Re}\left(\varepsilon_{k}\right)$ and $\operatorname{Im}\left(\varepsilon_{k}\right)$ are depicted as a function of the external field on the positive and negative current biases of $J= \pm 3 \times 10^{7} \mathrm{~A} / \mathrm{cm}^{2}$ in Fig. $4 \mathrm{a}-4 \mathrm{~d}$. The mode $1(k=1)$ which has the highest frequency corresponds to the excitation of the BPL, and the mode $2(k=2)$ and $3(k=3)$ are 

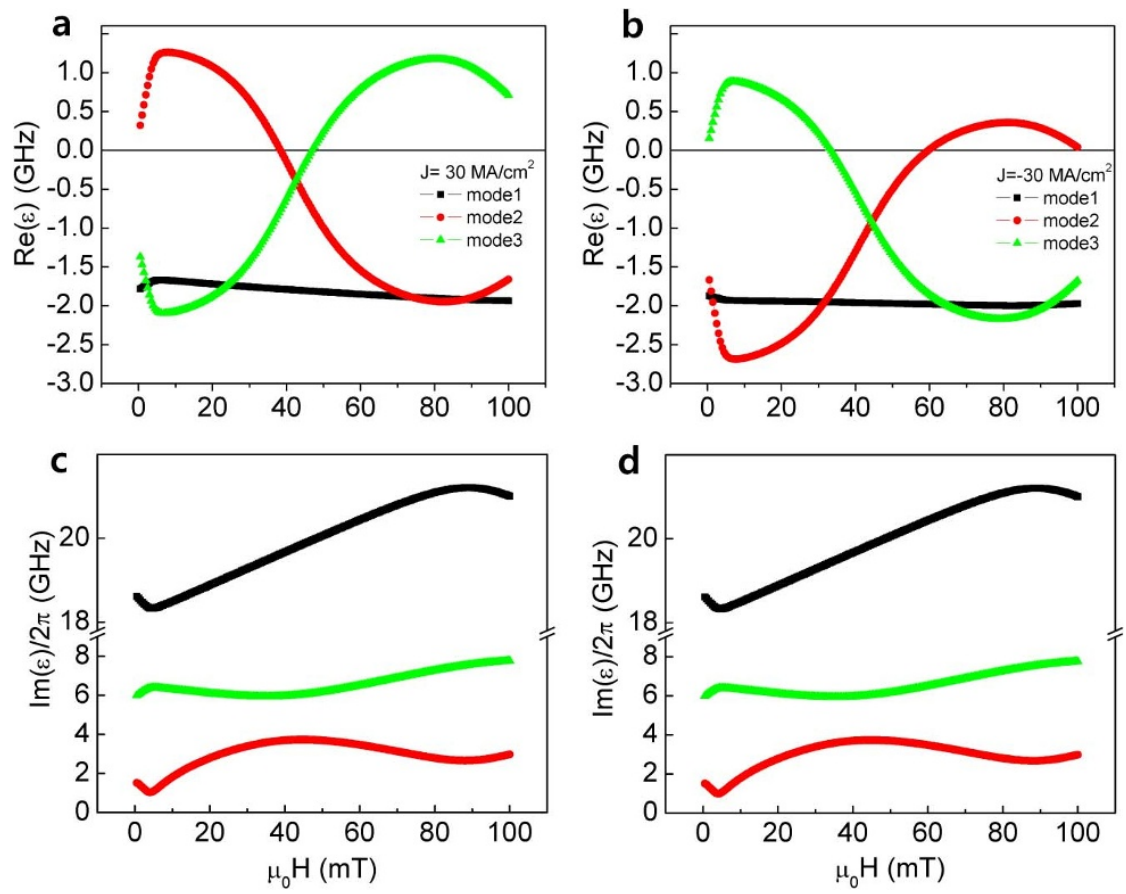

Figure $4 \mid$ Real parts of the eigenvalues of the three coupled magnetic layer system depicted as a function of the external field. (a),(b) Eigenvalues of all the three eigenmodes calculated under the positive and negative current biases of $J= \pm 3 \times 10^{7} \mathrm{~A} / \mathrm{cm}^{2}$, respectively. Mode 1 is the excitation of the BPL, and the mode 2 and 3 are respectively the optical and acoustic modes between the FL and TPL, which have been identified by the relative phase difference between the motions of the three layers. (a) The dominant precession mode where $\operatorname{Re}(\varepsilon)$ is positive changes from the mode 3 (acoustic) to the mode 2 (optical) at around $45 \mathrm{mT}$ as the field goes higher. (b) The positions of the dominant precession modes are reversed.

respectively the optical and acoustic modes between the FL and TPL. One can note that the optical mode has the higher frequency than the acoustic mode because the optical mode has the more dipolar energy. Each mode has been identified through the corresponding eigenvector as described in the supplementary information A.

Considering that the spin oscillation is the more heavily excited in the mode where $\operatorname{Re}\left(\varepsilon_{k}\right)$ is the larger, the eigenmode with the maximum $\operatorname{Re}\left(\varepsilon_{k}\right)$ among the three modes corresponds to the dominant spin precession mode. The dominant precession mode is the acoustic (optical) mode at a lower (higher) field of $\mu_{0} H<30 \mathrm{mT}\left(\mu_{0} \mathrm{H}>\right.$ $60 \mathrm{mT}$ ) on the case of positive current bias. On the negative current bias, it is reversed, i.e., the optical mode is dominant at the lower field and the acoustic mode is dominant at the higher field. Compared to the coupled motion of the FL and TPL, the motion of BPL is negligible. Although BPL is strongly coupled to the TPL by antiferromagnetic exchange coupling, the dipolar field is not fully compensated due to the different dynamic motions in the TPL and BPL. This reveals that the dipolar interaction should not be neglected in the dynamic case.

The eigenvalue of such a dominant precession mode under several different current densities is depicted as a function of the external field in Fig. 5. The real parts (imaginary parts) on the positive and negative current biases are shown in Fig. $5 \mathrm{a}$ and $5 \mathrm{~b}$ (in Fig. $5 c$ and $5 d$ ), respectively. The real part representing the decay rate of the oscillation becomes smaller and goes lower than zero around the field $45 \mathrm{mT}$ in both current polarities. This agrees with the experimental results that the steady state oscillation breaks down around $45 \mathrm{mT}$. The higher current density lifts up the $\operatorname{Re}(\varepsilon)$ values, which can be understood as the active reduction of the magnetic damping by the current. However, such a general behavior does not occur around the breakdown field, where the steady spin precession is inhibited no matter how high the current density is.

The imaginary part depicted in Fig. $5 c$ and $5 d$ shows a frequency gap around the breakdown point of $45 \mathrm{mT}$. The dominant oscillation mode changes from the acoustic (optical) mode to the optical (acoustic) mode with the increasing field in the positive (negative) current bias as depicted in Fig. 5c (Fig. 5d).

In order to investigate the effect of the dipolar coupling strength, we estimated the $\operatorname{Re}(\varepsilon)$ and $\operatorname{Im}(\varepsilon)$ values of the dominant precession mode for several different $s$ values. The results of $\operatorname{Re}(\varepsilon)$ as a function of external field are shown in Fig. 6a. Figure $6 \mathrm{~b}$ shows the dependence of the frequency gap upon the $s$ values, which was obtained from the results of $\operatorname{Im}(\varepsilon)$. The strong reduction of $\operatorname{Re}(\varepsilon)$ around the breakdown field observed at small $s$ becomes weaker and weaker as $s$ increases, resulting in a negligible dip of $\operatorname{Re}(\varepsilon)$ at $s=1000 \mathrm{~nm}$. The case of $s=$ $1000 \mathrm{~nm}$ can be considered as a system where each layer is coupled to the others only through the spin transfer torque without any dipolar interactions. In this case, we could not observe any special feature on $\operatorname{Re}(\varepsilon)$. This reveals that the anomalous magnetic damping observed around the breakdown field is strongly related to the dipolar coupling. The coupling only through the spin transfer torque cannot make such a behavior.

Recent micromagnetic calculations on a similar spin system have considered the dynamic coupling only by the spin transfer torque without any dipolar interaction ${ }^{16,17}$. Unlike our results, they showed several anomalous features such as the reduction of the spectral line-width and frequency jump exactly at the same breakdown field. But this behavior was observed only at a large current density $J>4.0 \times 10^{7} \mathrm{~A} / \mathrm{cm}^{2}$. The estimation of eigenvalues and eigenmodes assumes a spin motion with small amplitude around the stationary points. It cannot cover the dynamics of large amplitude. In this sense, the anomalous behaviors in purely spin transfer torque coupled system must come from the nonlinear dynamics in large amplitude. In fact, we carried out micromagnetic calculation at large current density, and it showed the same behaviors as the previous reports as described in the supplementary information B. The dipolar-coupling affect the damping property even at small spin amplitudes, making the anomalous damping behavior be observed experimentally in moderate current range. In contrast, the anomalous effect by the dynamic coupling 

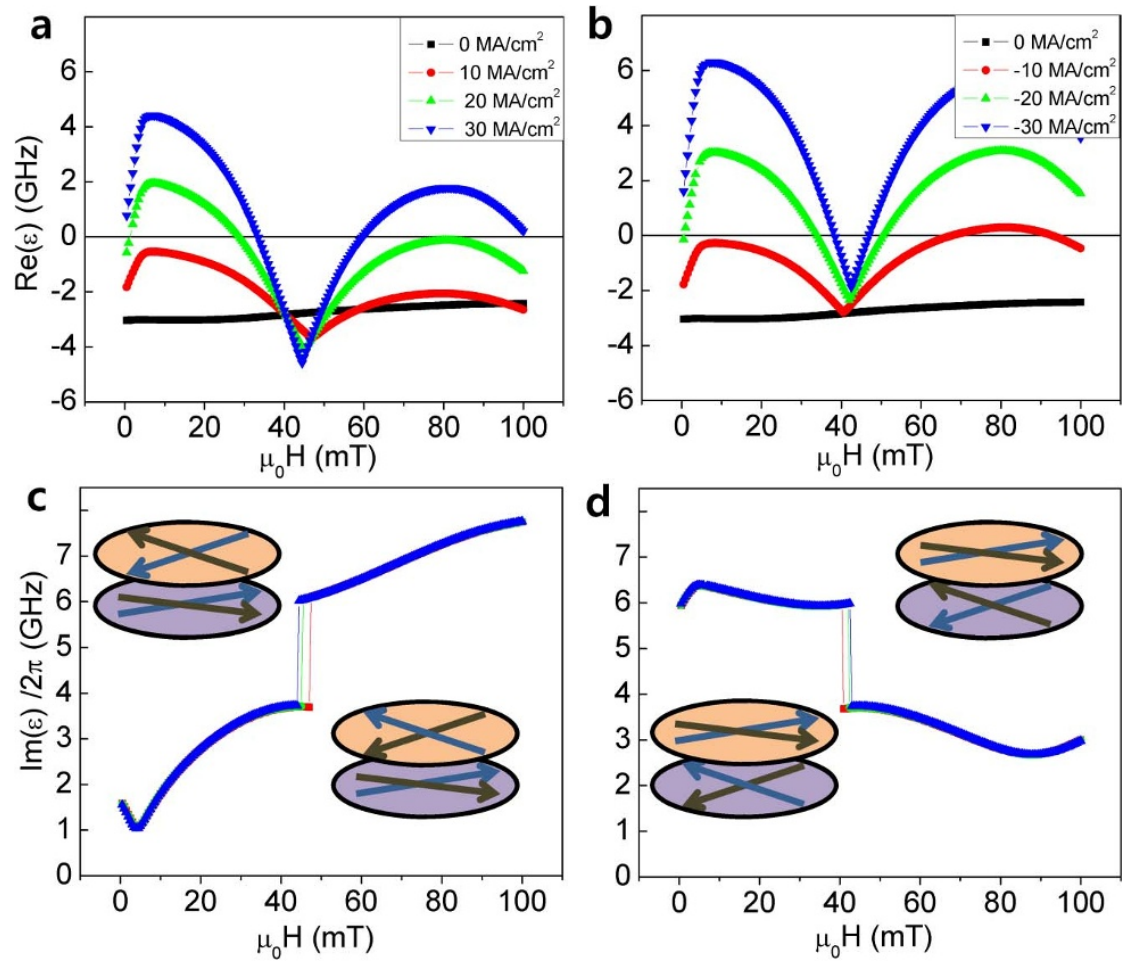

Figure 5 Real and imaginary parts of the eigenvalue of the coupled spin motion in azimuthal angle on different current densities. The eigenvalues $(\varepsilon)$ and eigenvectors of the coupled spin motion in azimuthal angle have been obtained with the method described in the text. Among the eigenvalues, the eigenvalue whose real part is the largest is plotted as a function of external field in the case of $s=0.8 \mathrm{~nm}$. (a),(b) Real part Re( $(\varepsilon)$ calculated on the positive and negative current biases, respectively. $\operatorname{Re}(\varepsilon)$ has a tendency to increase with the increasing current densities. However, it becomes smaller and goes below zero around $45 \mathrm{mT}$ irrespective of the current densities and polarities. (c),(d) Imaginary part $\operatorname{Im}(\varepsilon)$ calculated on the positive and negative current biases, respectively. $\operatorname{Im}(\varepsilon)$ shows similar frequency gap as the experiments and micromagnetic calculations. The relative phase of the spin oscillation between the FL and TPL has been identified through the eigenvectors. The schematic diagrams of the corresponding coupled spin motion are depicted in the inset of (c) and (d).

through the mutual spin transfer torque appears only at large spin amplitude.

Damping control through external magnetic field. According to the behavior of $\operatorname{Re}(\varepsilon)$ shown in Fig. $5 a$ and $5 b$, the damping of the coupled spin oscillation can be controlled by the external magnetic field. The real time motions of the spins in the FL and TPL have been obtained from the numerical calculation of Eq. 1 under the following condition. Initially the model system was under the field of $27 \mathrm{mT}$ far smaller than the breakdown point in the current bias of $J=+$ $2.0 \times 10^{7} \mathrm{~A} / \mathrm{cm}^{2}$. At the time $\tau=0 \mathrm{~ns}$, the magnetic field suddenly jumped from $27 \mathrm{mT}$ to the breakdown field. The azimuthal angle motions of the spins in the FL and TPL have been calculated in different $s$ values of $0.8 \mathrm{~nm}$ and $20 \mathrm{~nm}$. Figure 7a shows the results obtained on the case of $s=0.8 \mathrm{~nm}$. To compare the results with the eigenmodes that is valid only small spin oscillation limit, the results at a larger $\tau$ are separately depicted in Fig. $7 \mathrm{~b}$. Figure $7 \mathrm{c}$ and $7 \mathrm{~d}$ show the results similarly obtained on the case of $s=20 \mathrm{~nm}$.

As expected from the $\operatorname{Re}(\varepsilon)$ behavior shown in Fig. 5 and Fig. 6, the steady state precession below 0 ns decays out above 0 ns. Comparing the decaying region $(\tau>0)$ with the steady precession region $(\tau<0)$, one can find that the amplitude ratio between the FL and TPL is not
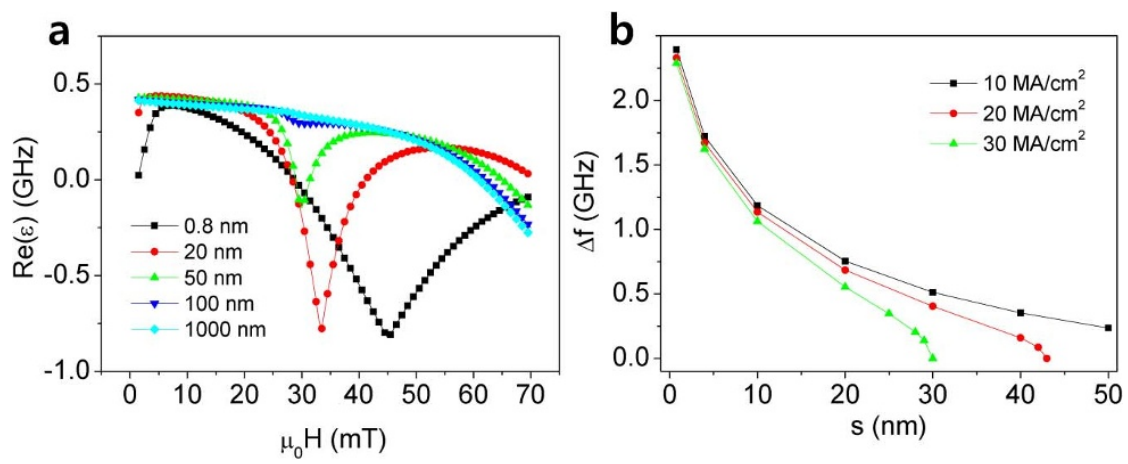

Figure 6 Effect of the dipolar interaction on $\operatorname{Re}(\varepsilon)$ and $\operatorname{Im}(\varepsilon)$ in dominant precession mode. (a) $\operatorname{Re}(\varepsilon)$ of the dominant precession mode depicted as a function of the external magnetic field under several different $s$ values. The strong reduction of $\operatorname{Re}(\varepsilon)$ around the breakdown field observed at small $s$ becomes weaker and weaker as $s$ increases, resulting in a negligible dip of $\operatorname{Re}(\varepsilon)$ at $s=1000 \mathrm{~nm}$. (b) Frequency gap $\Delta \mathrm{f}$ obtained from $\operatorname{Im}(\varepsilon)$ difference between the acoustic and optical mode at the breakdown point depicted as a function of $s$. 

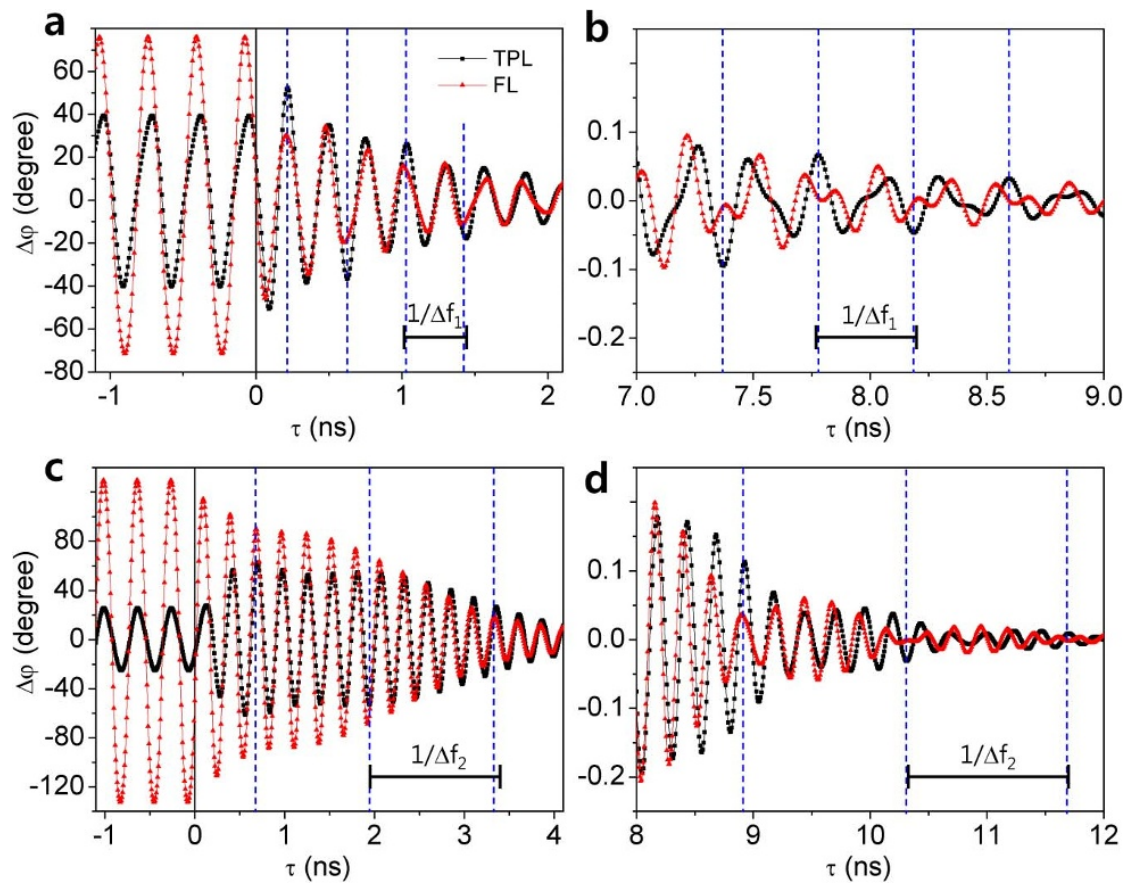

Figure $7 \mid$ Real time motion of the spins calculated in case that the field suddenly changes to the breakdown point. (a), (b) The real time motions of the spins in top pinned layer (TPL: black rectangles) and the free layer (FL: red triangles) have been calculated under the field changes described in the text on the case of $s=0.8 \mathrm{~nm}$. To clarify the beating pattern of the spin oscillation at $\tau>0$, dashed blue lines are marked on the points where the ratio of TPL amplitude over the FL amplitude has a local maximum. A scale bar representing the inverse of the frequency jump $\Delta \mathrm{f}_{1}$ on the case $s=0.8 \mathrm{~nm}$ is drawn to be compared with the period of beating pattern. (c), (d) The results obtained similarly on the case of $s=20 \mathrm{~nm}$. $\Delta \mathrm{f}_{2}$ represent the frequency jump on the case of $s=20 \mathrm{~nm}$.

constant but changes rather periodically. Dashed blue lines marked on the points where the ratio of TPL amplitude over the FL amplitude has a local maximum show this clearly. This beating pattern becomes even clearer when the amplitude of spin oscillation is small enough at a larger $\tau$, as depicted in Fig. $6 \mathrm{~b}$ and $6 \mathrm{~d}$. The period of beating becomes longer as the distance between the FL and TPL becomes larger as one can compare Fig. $6 \mathrm{~d}$ with Fig. $6 \mathrm{~b}$. For the comparison of the beating frequency with the frequency gap of the eigenmodes, scale bars of $1 / \Delta f_{1}$ and $1 / \Delta f_{2}$ have been drawn in Fig. 7 , which represent the inverses of the frequency gaps $\Delta \mathrm{f}_{1}$ and $\Delta \mathrm{f}_{2}$ obtained respectively from the $\operatorname{Im}(\varepsilon)$ values on the two cases $s=0.8 \mathrm{~nm}$ and $20 \mathrm{~nm}$ in Fig. 6b. One can find that the beating frequency identified by the dashed blue lines coincides with the frequency gap, i.e., the frequency difference between optical and acoustic mode at the breakdown field. This clearly reveals that the beating pattern comes from the interference of the two eigenmodes. Considering that the spin oscillation in the FL or TPL is expressed as a superposition of the acoustic and optical modes by Eq. 4, the observed beating pattern is not surprising. The beating pattern appears only near the breakdown field because the real part eigenvalues $\operatorname{Re}(\varepsilon)$ of the two competing modes are comparable only at this point. Away from this field, the difference of $\operatorname{Re}(\varepsilon)$ values is so large that only one eigenmode appears dominantly. In this sense, the increased damping at the breakdown point is closely related to the beating of the two eigenmodes.

Since the amplitude of the spin oscillation represents the energy stored in the spin motion, the beating pattern of the spin oscillation in each layer implies the energy transfer from the FL to TPL, and vice versa. In the field away from the breakdown point, both amplitudes of the FL and TPL remain the same without any energy transfer between the two layers. In the breakdown point, on the other hand, the FL and TPL change their amplitudes alternately, exchanging energies with each other.

Recent study on the two dipolar coupled magnetic disks has also shown similar energy transfer between the two disks ${ }^{30}$. The energy transfer in dipolar coupled system is in itself lossless and has no relation with the damping of the system. However, if the spin transfer torque is involved in the dipolar coupled spin layers like our sample, the energy transfer between the coupled layers affects the damping property. The current driven active damping on one layer is reversed on the other layer. If the FL is excited or anti-damped by the current bias, the TPL is the more effectively damped by the same current. Thus the transferred energy does not fully come back to the original layer due to the intrinsic and current driven active damping of the other layer. The energy transferred from one layer with a steady state spin precession supported by the current driven active anti-damping is more heavily dissipated by the active damping on the other layer. A steady spin precession is impossible if only the energy is transferred to the other layer. This is the case observed on the breakdown field. In spintronic devices like MRAM and STNO, the spin transfer torque is considered only to excite the spin state by the active anti-damping. But this is true only when the energy exchange between the FL and TPL is negligible. At the breakdown field, the FL transfers its energy to the TPL through the dipolar coupling, and the current driven spin precession ceases to exist.

\section{Discussion}

To summarize, we observed an anomalous breakdown of the current driven spin oscillation in the two spin layers, each of which is antiferromagnetically coupled to the other by the dipolar interaction, and one of which has an additional exchange bias field. From the micromagnetic calculations, the mechanism of the enhanced damping could be understood as the energy transfer between the two layers and the spin transfer torque acting reversely on each other. This damping mechanism gives another way to control the magnetic damping. We can control the damping of the coupled system by changing the resonant frequencies of the layers, i.e., by changing the external field. 
Besides, the magnetic field acts as a gate to control the energy transfer between the two layers. When the field is away from the breakdown point, the oscillation energies in the two layers are rather isolated. But by putting the field on the breakdown point, the energy flows to the other layer.

\section{Methods}

Sample Preparations. The multilayer film has been grown in the sputtering system with the base pressure around $10^{-7} \mathrm{~Pa}$ and post-annealed at $275^{\circ} \mathrm{C}$ under a magnetic field of about $1 \mathrm{~T}$ for two hours in order to align the spin orientations of the pinned layers in a specific direction. The sample has been patterned into a cylindrical nanopillar structure with a diameter of $90 \mathrm{~nm}$ by the electron beam lithography followed by ion beam etching. Subsequently, the sample was completely covered by the thick $\mathrm{SiO}_{2}$ film followed by the chemical mechanical polishing (CMP) to open only the top contact area. The bottom electrode contact area was patterned through the photolithography and ion beam etching up to the bottom layer (TiN). After opening the top and bottom electrode contact through an additional photolithography, the $\mathrm{Ti} / \mathrm{Au}$ electrodes have been formed through lift-off process. The bottom and top electrode have been formed in a form accessible by GSG type radio-frequency $(\mathrm{RF})$ probe.

Micromagnetic calculations. In the micromagnetic calculation, we did not take into account the spin transfer torque between the TPL and BPL. For the calculation of the microwave power shown in Fig. 3, the micromagnetic calculation was integrated with a model circuit that mimics the realistic measurement circuit. The calculation was carried out with the single domain model for each layer, but no significant changes were observed from the results using multi-domain model, because the applied current density through the MTJ is low enough. The following parameters were used for the MTJ: Layer structure is PtMn layer/ BPL(1.5 nm)/ Ru(0.8 nm)/ TPL(1.5 nm)/ $\mathrm{MgO}(0.8 \mathrm{~nm} \sim 1000 \mathrm{~nm}) / \mathrm{FL}(2.0 \mathrm{~nm})$. The layer has a circular shape with diameter $100 \mathrm{~nm}$. The material parameters are as follows. The saturation magnetization $M_{s}=$ $1,300 \mathrm{emu} / \mathrm{cc}$, perpendicular interface anisotropy $K_{s}=1.0 \mathrm{~mJ} / \mathrm{m}^{2}$ for FL, $M_{s}=$ $1240 \mathrm{emu} / \mathrm{cc}, K_{s}=0.9 \mathrm{~mJ} / \mathrm{m}^{2}$ for TPL, and $M_{s}=1430 \mathrm{emu} / \mathrm{cc}$, exchange bias field from the PtMn layer $\mu_{0} H_{e x}=150 \mathrm{mT}$ were used for BPL. Interlayer exchange coupling constant between TPL and BPL is $-1.3 \mu \mathrm{J} / \mathrm{m}$, damping constant $\alpha=0.01$, spin polarization $P=0.4$, TMR $=30 \%$, and $R A$ value in parallel state of $10 \mathrm{ohm} \mu \mathrm{m}^{2}$ were used. All parameters were chosen to mimic the experiments. In-plane external fields were applied along the easy axis with a tilt angle of $10^{\circ}$. Positive external fields favor the anti-parallel configuration of magnetizations. For the stochastic calculation, the Gaussian-distributed random fluctuation fields (mean $=0$, standard deviation $=$ [ $\left.2 k_{B} T /\left(M_{S} V \Delta t\right)\right]$, where $\Delta t$ is the integration time step, $V$ is the volume of unit cell $)^{31}$ have been added to the effective fields of LLGS equation.

1. Krivorotov, I. N. et al. Time-Domain Measurements of Nanomagnet Dynamics Driven by Spin-Transfer Torques. Science 307, 228-231 (2005).

2. Berger, L. Emission of spin waves by a magnetic multilayer traversed by a current. Phys. Rev. B 54, 9353-9358 (1996).

3. Slonczewski, J. C. Current-driven excitation of magnetic multilayers. J. Magn. Magn. Mater. 159, L1-L7 (1996).

4. Meyers, E. B. et al. Current-Induced Switching of Domains in Magnetic Multilayer Devices. Science 285, 867-870 (1999).

5. Sun, J. Z. Current-driven magnetic switching in manganite trilayer junctions. J. Magn. Magn. Mater. 202, 157-162 (1999).

6. Liu, Y. et al. Current-induced magnetization switching in magnetic tunnel junctions. Appl. Phys. Lett. 82, 2871-2873 (2003).

7. Katine, J. A. et al. Current-Driven Magnetization Reversal and Spin-Wave Excitations in Co/Cu/Co Pillars. Phys. Rev. Lett. 84, 3149-3152 (2000)

8. Tsoi, M. et al. Generation and detection of phase-coherent current-driven magnons in magnetic multilayers. Nature 406, 46-48 (2000).

9. Kiselev, S. I. et al. Microwave oscillations of a nanomagnet driven by a spinpolarized current. Nature 425, 380-383 (2003).

10. Rippard, W. H., Pufall, M. R. \& Silva, T. J. Quantitative studies of spinmomentum-transfer-induced excitations in $\mathrm{Co} / \mathrm{Cu}$ multilayer films using pointcontact spectroscopy. Appl. Phys. Lett. 82, 1260-1262 (2003).
11. Urazhdin, S. et al. Current-Driven Magnetic Excitations in Permalloy-Based Multilayer Nanopillars. Phys. Rev. Lett. 91, 146803 (2003).

12. Fuchs, G. D. et al. Spin-transfer effects in nanoscale magnetic tunnel junctions. Appl. Phys. Lett. 85, 1205-1207 (2004).

13. Wolf, S. A. et al. Spintronics: A Spin-Based Electronics Vision for the Future. Science 294, 1488-1495 (2001).

14. Chappert, C., Fert, A. \& Van Dau, F. N. The emergence of spin electronics in data storage. Nature Mater 6, 813-823 (2007).

15. Villard, P. et al. A GHz Spintronic-Based RF Oscillator. IEEE Journal of solid-state circuits 45, 214 (2010).

16. Gusakova, D. et al. Linewidth reduction in a spin-torque nano-oscillator caused by non conservative current-induced coupling between magnetic layers. Appl. Phys. Lett. 99, 052501 (2011).

17. Gusakova, D. et al. Spin-polarized current-induced excitations in a coupled magnetic layer system. Phys. Rev. B 79, 104406 (2009).

18. Van den Berg, H. A. M. et al. GMR sensor scheme with Artificial Antiferromagnetic Subsystem. IEEE Trans. Magn. 32, 4624-4626 (1996).

19. Leal, J. L. \& Kryder, M. H. Spin valves exchange biased by $\mathrm{Co} / \mathrm{Ru} / \mathrm{Co}$ synthetic antiferromagnets. J. Appl. Phys. 83, 3720-3723 (1998).

20. Petit, S. et al. Spin-Torque Influence on the High-Frequency Magnetization Fluctuations in Magnetic Tunnel Junctions. Phys. Rev. Lett 98, 077203 (2007).

21. Deac, A. M. et al. Bias-driven high-power microwave emission from MgO-based tunnel magnetoresistance devices. Nature Phys 4, 803-809 (2008).

22. Sankey, J. C. et al. Measurement of the spin-transfer-torque vector in magnetic tunnel junctions. Nature Phys 4, 67-71 (2008).

23. Cornelissen, S. et al. Free layer versus synthetic ferrimagnet layer auto-oscillations in nanopillars processed from $\mathrm{MgO}$-based magnetic tunnel junctions. Phys. Rev. B 81, 144408 (2010).

24. Helmer, A. et al. Quantized spin-wave modes in magnetic tunnel junction nanopillars. Phys. Rev. B 81, 094416 (2010).

25. Pi, U.H. et al. The effect of the static magnetic susceptibility on the spin precession in $\mathrm{MgO}$ based magnetic tunnel junction. Appl. Phys. Lett. 98, 242509 (2011).

26. Kittel, C. Introduction to Solid State Physics, 7th ed., p. 505. (John Wiley \& Sons, Inc., New York, 1996).

27. Li, Z. \& Zhang, S. Magnetization dynamics with a spin-transfer torque. Phys. Rev. B 68, 024404 (2003).

28. Lee, K. J. et al. Excitations of incoherent spin-waves due to spin-transfer torque. Nature Mater 3, 877-881 (2004).

29. Boulle, O. et al. Shaped angular dependence of the spin-transfer torque and microwave generation without magnetic field. Nature Phys 3, 492-497 (2007).

30. Jung, H. et al. Tunable negligible-loss energy transfer between dipolar-coupled magnetic disks by stimulated vortex gyration. Scientific Reports 1, 59 (2011).

31. Brown, W. F. Thermal fluctuation of fine ferromagnetic particles. IEEE Trans. Magn. 15, 1196-1208 (1979).

\section{Author contributions}

S.C.Lee and U.H.Pi contributed equally to this work especially on the analysis. S.C.Lee executed the micromagnetic calculation based on LLGS equation. U.H.Pi carried out the measurements and wrote the manuscript. K.Kim designed and grew the magnetic film, and K.S.Kim fabricated the device and directed the project. J.Shin and U.Chung supervised all the works.

\section{Additional information}

Supplementary information accompanies this paper at http://www.nature.com/ scientificreports

Competing financial interests: The authors declare no competing financial interests.

License: This work is licensed under a Creative Commons

Attribution-NonCommercial-ShareAlike 3.0 Unported License. To view a copy of this license, visit http://creativecommons.org/licenses/by-nc-sa/3.0/

How to cite this article: Lee, S.C. et al. Current Driven Magnetic Damping in Dipolar-Coupled Spin System. Sci. Rep. 2, 531; DOI:10.1038/srep00531 (2012). 